\title{
The Zugspitze radiative closure experiment for quantifying water vapor absorption over the terrestrial and solar infrared - Part 2: Accurate calibration of high spectral-resolution infrared measurements of surface solar radiation
}

\author{
Andreas Reichert, Markus Rettinger, and Ralf Sussmann \\ Karlsruhe Institute of Technology, IMK-IFU, Garmisch-Partenkirchen, Germany \\ Correspondence to: Ralf Sussmann (ralf.sussmann@kit.edu) \\ Received: 13 April 2016 - Published in Atmos. Meas. Tech. Discuss.: 25 April 2016 \\ Revised: 30 August 2016 - Accepted: 2 September 2016 - Published: 21 September 2016
}

\begin{abstract}
Quantitative knowledge of water vapor absorption is crucial for accurate climate simulations. An open science question in this context concerns the strength of the water vapor continuum in the near infrared (NIR) at atmospheric temperatures, which is still to be quantified by measurements. This issue can be addressed with radiative closure experiments using solar absorption spectra. However, the spectra used for water vapor continuum quantification have to be radiometrically calibrated. We present for the first time a method that yields sufficient calibration accuracy for NIR water vapor continuum quantification in an atmospheric closure experiment. Our method combines the Langley method with spectral radiance measurements of a hightemperature blackbody calibration source $(<2000 \mathrm{~K})$. The calibration scheme is demonstrated in the spectral range 2500 to $7800 \mathrm{~cm}^{-1}$, but minor modifications to the method enable calibration also throughout the remainder of the NIR spectral range. The resulting uncertainty $(2 \sigma)$ excluding the contribution due to inaccuracies in the extra-atmospheric solar spectrum (ESS) is below $1 \%$ in window regions and up to $1.7 \%$ within absorption bands. The overall radiometric accuracy of the calibration depends on the ESS uncertainty, on which at present no firm consensus has been reached in the NIR. However, as is shown in the companion publication Reichert and Sussmann (2016), ESS uncertainty is only of minor importance for the specific aim of this study, i.e., the quantification of the water vapor continuum in a closure experiment. The calibration uncertainty estimate is substantiated by the investigation of calibration self-consistency, which yields compatible results within the estimated errors for $91.1 \%$ of the
\end{abstract}

2500 to $7800 \mathrm{~cm}^{-1}$ range. Additionally, a comparison of a set of calibrated spectra to radiative transfer model calculations yields consistent results within the estimated errors for $97.7 \%$ of the spectral range.

\section{Introduction}

Solar absorption spectra in the near-infrared (NIR, 4000$14000 \mathrm{~cm}^{-1}$ ) spectral domain contain a wealth of information on atmospheric radiative processes such as the absorption of radiation by atmospheric trace gases or scattering processes by clouds and aerosols. Measurements of solar absorption spectra can also be used to obtain better quantitative knowledge of the NIR absorption properties of the most important atmospheric greenhouse gas, i.e., water vapor. In addition to numerous absorption lines attributable to vibrationrotation transitions, in the case of water vapor the so-called continuum absorption has to be taken into account. The continuum absorption has significant effects on the atmospheric radiative budget (e.g., Paynter and Ramaswamy, 2011, 2014), and, consequently, knowledge of its exact magnitude is crucial for climate models (Turner et al., 2012; Rädel et al., 2015). The magnitude of the NIR water vapor continuum has been constrained in a number of laboratory experiments (e.g., Burch, 1982; Paynter et al., 2009; Ptashnik et al., 2011). Due to the decrease in water vapor saturation pressure with decreasing temperature and given the sensitivity of currently available experimental setups, such studies have been per- 
formed at least at room temperature or even heated to be able to measure the weak continuum absorption. Therefore, accurate laboratory studies have not yet been performed at atmospheric temperatures. However, the extrapolation of results to lower temperature leads to significant errors because the temperature dependence of continuum absorption is in general not well modeled (e.g., Paynter and Ramaswamy, 2011). Setting up new experiments performed in the real atmosphere rather than in the laboratory is therefore highly desirable.

In the far- and mid-infrared spectral range (FIR and MIR), a successful way to quantify the water vapor continuum at atmospheric temperatures has been demonstrated via socalled radiative closure experiments combining spectrally resolved atmospheric thermal emission measurements with coincident measurements of the atmospheric state (e.g., Tobin et al., 1999; Serio et al., 2008; Delamere et al., 2010). In the NIR, however, closure studies of this kind have not yet been performed because the atmospheric thermal emission is too weak in this spectral domain. A potential solution to this problem in the NIR is the use of solar absorption spectra. We therefore aim to perform a radiative closure experiment using solar absorption spectra at the Zugspitze $\left(47.42^{\circ} \mathrm{N}, 10.98^{\circ} \mathrm{E} ; 2964 \mathrm{~m}\right.$ a.s.1.) observatory. In the framework of the Network for the Detection of Atmospheric Composition Change (NDACC; Kurylo, 1991; http://www.ndacc. org/), solar Fourier transform infrared (FTIR) measurements are regularly carried out at the Zugspitze site (Sussmann and Schäfer, 1997). However, the application of solar absorption spectra requires the observations to be radiometrically calibrated. Currently, the standard solar FTIR instrumentations used within the networks NDACC and TCCON (Total Carbon Column Observing Network, http://www.tccon.caltech. edu/, Wunch et al., 2011) do not include radiometric calibration.

In the far- and mid-infrared spectral range, a calibration method for high-resolution spectral radiance measurements based on the observation of two blackbody sources at different temperatures is well established (Revercomb et al., 1988). However, there is currently no standard calibration scheme available for the NIR spectral range. Possible methods include the use of standard lamps (see, e.g., Schmid and Wehrli, 1995). Alternatively, Gardiner et al. (2012) implemented a calibration method based on spectral radiance measurements of a very high-temperature $(3000 \mathrm{~K})$ blackbody source. This method is traceable to a primary standard cryogenic radiometer, and a calibration transfer for field measurements was implemented via a portable calibration source (National Physical Laboratory (NPL) Transfer Standard Absolute Radiance Source, TSARS). This transfer of calibration for field measurements is of crucial importance because radiative closure experiments are typically carried out at remote (mountain or polar) observatories because of the low atmospheric humidity required. However, the installation of a very high-temperature calibration source is highly challenging at such sites for several reasons: many remote observato- ries, including the Zugspitze site, lack sufficient laboratory space with stable ambient conditions (especially temperature) for the installation of a very high-temperature blackbody. Accessibility of the site with heavy instruments may be a further restriction, as is the case for the Zugspitze observatory, where access is only possible by cable car. The calibration method proposed in this study offers an alternative approach to this issue and does not require access to a very high-temperature calibration source.

Furthermore, given the calibration accuracy of 3.3 to $5.9 \%$ attainable with the approach proposed by Gardiner et al. (2012), significant continuum absorption could only be measured in a small fraction of the 2500 to $7800 \mathrm{~cm}^{-1}$ spectral range with our experimental setup. More specifically, the range of integrated vertical water vapor column (IWV) covered by our data set is 1.4 to $3.3 \mathrm{~mm}$. Taking into account additional sources of radiance uncertainty in our closure setup (described in detail in the companion paper Sussmann et al., 2016, same issue, hereafter referred to as Part 1) and assuming the MT_CKD 2.5.2 continuum model (Mlawer et al., 2012), a calibration accuracy $<2 \%$ is necessary to measure significant continuum absorption throughout at least $33 \%$ of the 2500 to $7800 \mathrm{~cm}^{-1}$ spectral range covered by our measurements.

It is therefore the goal of this paper to demonstrate an alternative calibration scheme which overcomes these shortcomings and meets the calibration uncertainty of $<2 \%$ required for water vapor continuum quantification in the Zugspitze closure experiment. Our calibration strategy is a synergetic combination of two approaches. First, we use the Langley calibration method that allows for precise absolute calibration in selected spectral windows. Additionally, our calibration relies on spectral radiance measurements of a blackbody source $(1973 \mathrm{~K})$ to gain information on the shape of the calibration curve between these windows. The combined method provides sufficiently accurate radiometric calibration of NIR solar FTIR spectra to subsequently determine water vapor continuum magnitude and validate water vapor line parameters. Our approach eliminates the need for a very hightemperature source only available in few designated laboratories. This paper, hereafter referred to as Part 2, constitutes the second contribution in a trilogy of papers describing the Zugspitze radiative closure experiment. Part 1 introduces the setup of the experiment, provides a sensitivity analysis, and presents results on the far-infrared water vapor continuum. Part 3 (Reichert and Sussmann, 2016) describes the quantification of the NIR water vapor continuum based on the radiometric calibration method provided in this study.

Our paper is organized as follows. In Sect. 2, the instrumental setup of the Zugspitze solar FTIR spectrometer, the typical configuration of the spectral radiance measurements for which the calibration is applied, and the blackbody calibration source are presented. Section 3 gives a description of the combined calibration strategy, while the uncertainty associated with the calibration procedure is outlined in Sect. 4. 
Table 1. Instrumental specifications and settings chosen for the solar FTIR spectra acquisition.

\begin{tabular}{ll}
\hline Instrument type & Bruker IFS125HR \\
\hline Detector & InSb $\left(1850\right.$ to $\left.9600 \mathrm{~cm}^{-1}\right)$ or InGaAs $\left(4000\right.$ to $\left.12800 \mathrm{~cm}^{-1}\right)$ \\
Beam splitter & $\mathrm{KBr}$ or $\mathrm{CaF}_{2}$ \\
Number of scans per spectrum & four for Langley measurements, eight for further spectra \\
Spectral resolution & $0.02 \mathrm{~cm}^{-1}$ (where resolution is defined as 0.9/OPD) \\
FOV diameter & $0.07^{\circ}$ \\
Scan rate & $40 \mathrm{kHz}$ \\
Average signal-to-noise ratio & 172 (for Langley measurements) \\
\hline
\end{tabular}

In Sect. 5, the validation of calibration results is discussed. Finally, Sect. 6 provides a summary and conclusions.

\section{Instrumental setup}

\subsection{Zugspitze NIR solar absorption measurements}

The NIR solar absorption spectra to which the calibration is applied are measured with a solar FTIR spectrometer located at the high-altitude observatory on the summit of the Zugspitze, Germany (47.42 ${ }^{\circ} \mathrm{N}, 10.98^{\circ} \mathrm{E} ; 2964 \mathrm{~m}$ a.s.1.). The spectrometer setup is described in detail in Sussmann and Schäfer (1997). An overview of the FTIR spectra acquisition settings is provided in Table 1. Briefly, the measurements are obtained with a Bruker IFS125HR interferometer. The interferometer's field of view actively tracks the position of the center of the solar disc by means of feedback from a quadrant diode. Interferograms were recorded with a maximum optical path difference (OPD) of $45 \mathrm{~cm}$ and averaged over four to eight scans.

Spectra are typically measured with a liquid-nitrogencooled $\mathrm{InSb}$ detector $\left(1850\right.$ to $\left.9600 \mathrm{~cm}^{-1}\right)$ in combination with a $\mathrm{KBr}$ beam splitter. An alternative setup consisting of an InGaAs detector (4000 to $12800 \mathrm{~cm}^{-1}$ ) and a $\mathrm{CaF}_{2}$ beam splitter is available. This setup has the advantage of an improved signal-to-noise ratio, especially above $6000 \mathrm{~cm}^{-1}$, and avoids a spectral interval of low instrumental sensitivity between 5200 and $5800 \mathrm{~cm}^{-1}$ attributed to low transmissivity of the $\mathrm{KBr}$ beam splitter. However, for routine operations of the solar FTIR spectrometer, especially for the long-term determination of atmospheric trace gas column amounts and vertical profiles (e.g., Borsdorff and Sussmann, 2009; Sussmann et al., 2009, 2012; Hausmann et al., 2016), the first option is more suitable. Therefore, most spectra to be calibrated were recorded with the $\mathrm{InSb} / \mathrm{KBr}$ setup, while the $\mathrm{InGaAs} / \mathrm{CaF}_{2}$ setup was only used in a number of single-day measurement campaigns. While the calibration scheme presented here is applicable for both configurations, we therefore focus on the $\mathrm{InSb} / \mathrm{KBr}$ setup throughout the remainder of this paper.

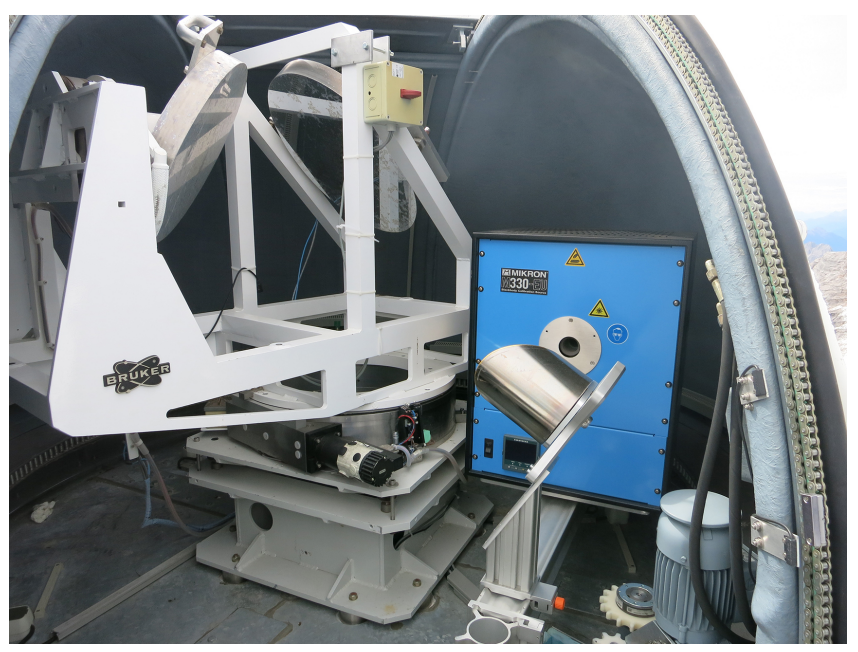

Figure 1. Blackbody calibration source inside the Zugspitze solar FTIR dome, additional $90^{\circ}$ off-axis mirror and solar tracker.

\subsection{Blackbody calibration unit}

The calibration procedure makes use of a blackbody calibration source installed in the Zugspitze solar FTIR dome. The calibration source (MIKRON M330-EU, Lumasense Technologies) is shown in Fig. 1, while its technical specifications according to the manufacturer are given in Table 2. Blackbody spectra are measured with the solar FTIR using the solar tracker optics and an additional gold-coated $90^{\circ}$ offaxis mirror. The thermal radiation emitted by the calibration source is directed to the solar tracker by means of the additional off-axis mirror, whose distance to the blackbody cavity interior is set to its focal length of $f=478 \mathrm{~mm}$. Blackbody source and off-axis mirror are aligned in order to produce a parallel beam with a diameter of $156 \mathrm{~mm}$ that is incident at a $90^{\circ}$ angle on the solar tracker elevation mirror.

\section{Calibration method}

Our approach comprises a Langley-type calibration described in Sect. 3.1 and a blackbody calibration presented in Sect. 3.2. Our new calibration strategy is a combination 
of both as explained in Sect. 3.3. The calibration procedure consists of deducing a calibration curve $c(v)$, which is finally multiplied with the measured spectra to achieve radiometric calibration.

\subsection{Langley calibration}

\subsubsection{General description}

The Langley method (e.g., Liou, 2002) has been frequently used for solar-constant determination or calibration of sun photometers. It relies on repeated measurements of solar irradiance or radiance at a range of solar zenith angles. According to the Beer-Bouguer-Lambert law, the direct solar irradiance at a wavenumber $v$ observed at the surface is given by

$F(v)=F_{0}(v) \cdot \exp (-k(v) \cdot m)$,

where $F_{0}(v)$ denotes the extra-atmospheric solar irradiance, $k(v)$ the atmospheric absorption coefficient, and $m$ the relative air mass, i.e., the ratio of the air mass along the line of sight of the observer to the sun and the air mass in zenith direction. Taking the logarithm of Eq. (1) results in the linear relation

$\ln (F(v))=\ln \left(F_{0}(v)\right)-k(v) \cdot m$.

Equation (2) implies that the logarithm of the extraatmospheric solar irradiance can be calculated by measuring solar irradiance in a range of air mass values and extrapolation of the results to air mass 0 according to the best-fit linear relation between air mass and logarithm of measured irradiance. The resulting value of $F_{0}(v)$ can then be compared to the known extra-atmospheric solar spectrum (ESS) to yield a radiometric calibration at that wavenumber. An inversion of this scheme, i.e., deducing an ESS from calibrated solar FTIR measurements was presented by Menang et al. (2013).

Equation (2) is only fulfilled if atmospheric properties like IWV or aerosol optical thickness do not vary during the measurements. Since IWV varies significantly even on the timescale of a few hours (e.g., Kämpfer et al., 2013; Vogelmann et al., 2015), accurate Langley measurements in spectral regions with significant absorption by water vapor have to be carried out within short time intervals, i.e., at high solar zenith angles. We thereby limited the duration of Langley measurements to 1-2 h, which, based on the results of Vogelmann et al. (2015), leads to an IWV variability of about $1 \mathrm{~mm}$ during the measurements. Refraction has a significant influence at high solar zenith angles. In order to include refraction effects, air mass values used in this study were computed by means of ray tracing calculations. More specifically, the ray tracing routine of the PROFFIT software (Hase et al., 2004) was used for air mass calculation. Since atmospheric absorption is dominated by water vapor for most spectral points considered in this study, instead of using the air column, the

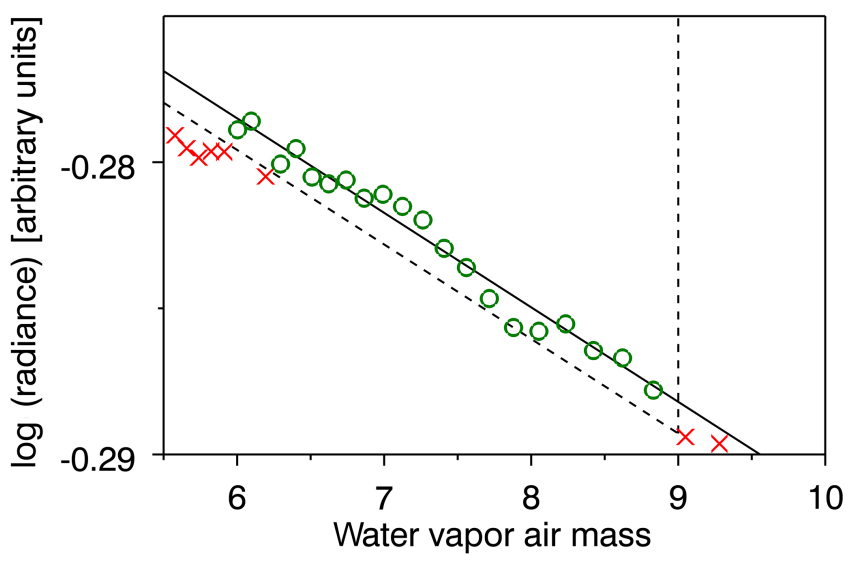

Figure 2. Selection of spectra for the Langley measurements made on 13 December 2013. The Langley plot is based on radiance measurements in the 4300 to $4350 \mathrm{~cm}^{-1}$ range. Continuous black line: first-estimate linear fit; dashed black line: selection threshold; green circles: selected spectra; red crosses: excluded spectra.

related water vapor column was utilized as an air mass input to the Langley fits.

\subsubsection{Selection of suitable spectra}

Langley calibration coefficients were determined from daily sets of selected solar FTIR spectra recorded under apparently cloud-free conditions at $0.02 \mathrm{~cm}^{-1}$ resolution (where resolution is defined as 0.9 /OPD). Only spectra averaged over four scans were used for the Langley fit to reduce air mass and atmospheric-state variation during the spectral averaging time interval. Several effects may lead to radiance measurements inconsistent with Eq. (2) and thereby lead to biased calibration results. A first issue is related to the fact that the instrument's field of view (FOV) covers different areas of the solar tracker mirrors throughout the day. This is a result of inaccuracies in mirror alignment. Mirror aging and dirtying lead to spatially inhomogeneous reflectivity of the tracker mirrors. Therefore, even if the atmospheric radiance is constant, a spurious variation in measured radiance depending on the instrument's viewing direction can be detected, which leads to calibration errors. Temporal variation in thin cloud cover in the line of sight of the solar FTIR leads to variations in the measured radiance and therefore also biases the calibration result.

We use a preliminary Langley plot to select the spectra which are least affected by cloud, IWV variation, and FOV effects:

i. A spectral interval with little molecular absorption, namely $4300 \mathrm{~cm}^{-1}<v<4350 \mathrm{~cm}^{-1}$, was selected. Within this interval, Langley plots according to Eq. (2) were generated using the mean radiance for spectral points with the lowest atmospheric optical depth (red and green points in Fig. 2). 
ii. A first estimate of the linear relation avoiding cloud and FOV bias (continuous black line in Fig. 2) was fitted using the spectra with the highest mean radiance within each air mass bin (width $\Delta m=1$ ). Air mass bins for which the mean radiance did not decrease with increasing air mass as expected for cloud-free measurements were discarded.

iii. The maximum deviation from the ideal linear relation not attributable to FOV influence or IWV temporal variability was calculated. The FOV effect was estimated as outlined in Sect. 4.1. The IWV influence was estimated based on the expected variability of about $1 \mathrm{~mm}$ during the 1-2 $\mathrm{h}$ Langley measurements according to Vogelmann et al. (2015).

iv. Spectra consistent with the linear relation determined in (ii) minus the maximum deviation estimated in (iii) measured at an air mass less than 9.0 were selected for further analysis (dashed line in Fig. 2). An air mass threshold is required since, at very high solar zenith angles, air mass calculation becomes increasingly inaccurate, and air mass changes significantly during the spectral averaging period. The air mass threshold of 9.0 was chosen because beyond this value, significant deviations from the linear relation according to Eq. (2) can be observed, which indicate inaccuracies in the air mass calculation. The selected spectra are shown as green circles in Fig. 2, while discarded spectra are shown in red.

We further processed Langley data sets with a sufficiently high number of selected spectra according to the selection criteria presented above ( $>10$ measurements) over an air mass range of at least $\Delta m=2$. The uncertainty of the Langley fit increases with decreasing number of spectra, and the threshold for the minimum number of spectra was adjusted in order to achieve a Langley uncertainty comparable with the other contributions to the calibration uncertainty budget (see Fig. 6). Dry atmospheric conditions increase the fraction of spectral intervals suitable for accurate calibration and generally reduce the Langley fit uncertainty due to lower atmospheric optical depth. Therefore, only Langley measurements with IWV $<5 \mathrm{~mm}$ were included in the analysis. While sufficiently dry atmospheric conditions occur at the Zugspitze site year-round (see Fig. 3 of Part 1), due to instrumentation availability issues, Langley measurements fulfilling the selection criteria were only recorded on 12 and 13 December 2013.

\subsubsection{Langley fit}

A number of issues have to be considered before generating Langley fits according to Eq. (2) using the selected spectra. During measurements, the line of sight of the solar FTIR continuously tracks the position of the center of the solar disc. Sun tracking inaccuracies influence the Langley measurements due to the fact that the FOV of the solar FTIR only

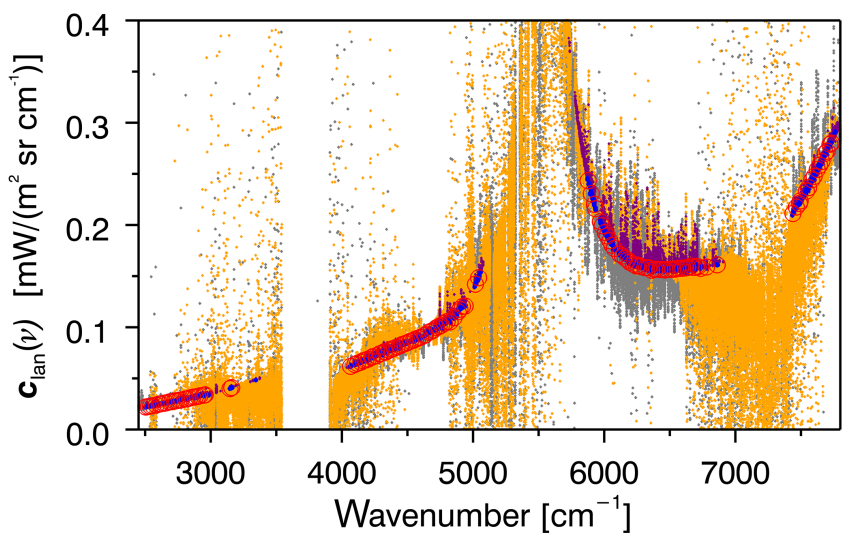

Figure 3. Selection of suitable spectral points and averaging for Langley calibration (measurements from 13 December 2013). Grey: initial Langley fit results; orange: results not affected by solar lines; purple: results after applying fit uncertainty threshold; blue: final results after applying stability threshold; red circles: Langley calibration results averaged over $20 \mathrm{~cm}^{-1}$ bins.

covers a fraction of the solar disc (FOV diameter for Langley measurements $0.07^{\circ}$ ) and the spatial inhomogeneity of the solar emission, the so-called solar limb darkening. Furthermore, mispointing leads to erroneous air mass input for the Langley fit. As outlined in Reichert et al. (2015), systematic mispointing can be determined using multiple measurements of solar line Doppler shifts at different orientations of the solar rotation axis. Using this method, mispointing-corrected air mass values can be calculated as input to the Langley fits. The effect of solar limb darkening can be corrected for using the analytical description of relative intensity of NIR solar radiation depending on the fractional radius $r$ given by Hestroffer and Magnan (1998),

$I(r)=\left(1-r^{2}\right)^{\alpha / 2}$,

where $\alpha=-0.023+0.292 \times 10^{-4} \mathrm{~cm} \times v$. The mispointing correction consists of multiplying the calibration result by $I(r)$, where $r$ designates the norm of the mispointing divided by the apparent solar radius.

\subsubsection{Choice of ESS}

The Langley calibration method requires knowledge of the ESS. In this study, we use the semiempirical synthetic ESS of Kurucz (2005). This extra-atmospheric spectrum is widely adopted for atmospheric radiative transfer calculations due to its high spectral resolution (radiance spectra are provided with $0.1 \mathrm{~cm}^{-1}$ point spacing) and its wide spectral range ( 0 to $50000 \mathrm{~cm}^{-1}$ ). The ESS was scaled to account for the Earth's orbital eccentricity. Alternative ESS data can be used for the Langley calibration without further modification of the calibration scheme. Suitable choices include, e.g., the ESS proposed by Thuillier et al. (2003) that is based on satellite observations. This spectrum covers a range of 0.1 to $2400 \mathrm{~nm}$ 
with an NIR spectral resolution of $0.5 \mathrm{~nm}$. This resolution allows for the identification of broad solar lines but does not provide fine enough resolution for a detailed representation of solar line shapes. An advantage of the Thuillier et al. (2003) solar spectrum over the Kurucz (2005) spectrum is that it includes an uncertainty estimate that can be used in the uncertainty analysis of the radiometric calibration provided in Sect. 4.2. Alternative satellite-based ESS versions have been presented by Thuillier et al. (2014, 2015). Further alternatives include solar spectra derived from surface observations, such as the high-resolution ESS by Menang et al. (2013) that covers the $4000-10000 \mathrm{~cm}^{-1}$ spectral range and was deduced via the Langley method from solar FTIR spectra radiometrically calibrated with the method by Gardiner et al. (2012). An important advantage of the ESS by Menang et al. (2013) is that it includes numerous solar spectral lines omitted in the ESS by Kurucz (2005). Recent ESS studies have yielded differences in the infrared solar radiance of 5-10\%, which partly exceed the respective uncertainty estimates. The impact of these recent results on the calibration accuracy is discussed in Sect. 4.2.

\subsubsection{Spectral window selection}

The uncertainty of the Langley calibration varies strongly throughout the spectrum. Therefore, it is necessary to select spectral windows in which accurate Langley results can be obtained. Figure 3 shows the selection steps applied to the Langley calibration results $c_{\text {lan }}(v)$. In the following, several selection criteria make use of upper or lower linear envelopes to the spectra. These envelopes are constructed by selecting the highest or lowest values in the spectra within each wavenumber bin of a given width. The envelope is then constructed by linear interpolation between these selected points. Specifically, the following selection criteria were used:

i. Spectral points within solar lines are excluded due to the higher ESS radiance uncertainty in these regions. More specifically, all points with ESS radiance more than $1 \%$ below the upper linear envelope using $20 \mathrm{~cm}^{-1}$ width bins are excluded (grey points in Fig. 3).

ii. All spectral points for which the relative Langley fit uncertainty was above $0.4 \%$ were discarded (orange points in Fig. 3).

iii. Furthermore, regions within solar lines not included in the ESS of Kurucz (2005) and points with spurious low fit uncertainty due to radiance measurement noise were excluded. For this purpose, all points for which the standard deviation of Langley calibration results within a $0.1 \mathrm{~cm}^{-1}$ wide interval around any given spectral point exceeds $0.3 \%$ were excluded (purple points in Fig. 3).

Blackbody spectral radiance measurements (see Sect. 3.2) show that the solar FTIR calibration curve varies only slowly
Table 2. Specifications of the blackbody calibration source.

\begin{tabular}{ll}
\hline Type & MIKRON M330-EU \\
\hline Manufacturer & Lumasense Technologies \\
Temperature range & 573.15 to $1973.15 \mathrm{~K}$ \\
Temperature uncertainty & $\pm 0.025 \cdot(T-273.15 \mathrm{~K}) \pm 1 \mathrm{~K}$ \\
Source homogeneity & $\pm 1 \mathrm{~K}$ within inner $1 / 3$ of aperture \\
Aperture diameter & $25 \mathrm{~mm}$ \\
Emissivity & $0.99 \pm 0.005$ \\
\hline
\end{tabular}

with wavenumber. The filtered Langley results (blue points in Fig. 3) were therefore averaged (error-weighted mean using Langley fit uncertainties) over $20 \mathrm{~cm}^{-1}$ windows in order to further reduce statistical uncertainty. The final averaged Langley calibration coefficients are shown as red circles in Fig. 3 and are designated "Langley points" throughout the remainder of this study.

\subsection{Blackbody radiance measurements}

Spectral radiance measurements of the blackbody calibration source described in Sect. 2.2 are used to determine the shape of the calibration curve in spectral intervals between the points suitable for precise Langley calibration. Contrary to the calibration approach described by Gardiner et al. (2012), using a source with a temperature of $3000 \mathrm{~K}$, a lower cavity temperature of $1973.15 \mathrm{~K}$ can be used in the Zugspitze experiment due to the combination with Langley measurements. The settings of the FTIR spectra acquisition were similar to the Langley measurements (see Table 2).

As for the Langley measurements, dry atmospheric conditions imply more narrow spectral intervals affected by water vapor line absorption and thereby improve the blackbody calibration accuracy. Therefore, only measurements with an atmospheric water vapor density $\rho_{\mathrm{H}_{2} \mathrm{O}}<1 \mathrm{~g} \mathrm{~m}^{-3}$ at the Zugspitze summit observatory were considered for calibration. The blackbody thermostat generally compensates for the effect of changing air temperature inside the FTIR dome to keep the temperature inside the blackbody cavity constant and thereby avoid temporal variation of the emitted blackbody radiance. This is demonstrated in the red line in Fig. 6, which corresponds to 2 times the standard deviation of all normalized blackbody calibration curves. The measurements show that within a range of ambient temperatures from 263 to $273 \mathrm{~K}$, the blackbody calibration results show only very little variation. However, fast temporal variations in air temperature cannot be compensated for by the blackbody thermostat instantaneously and lead to short time intervals with temporally unstable blackbody radiance. Such measurements were discarded before further analysis.

Only spectral points outside water vapor lines were considered to avoid bias in the calibration. More specifically, only points less than $10 \sigma$ (where $\sigma$ designates the mean measurement noise) below the upper envelope of the measured 


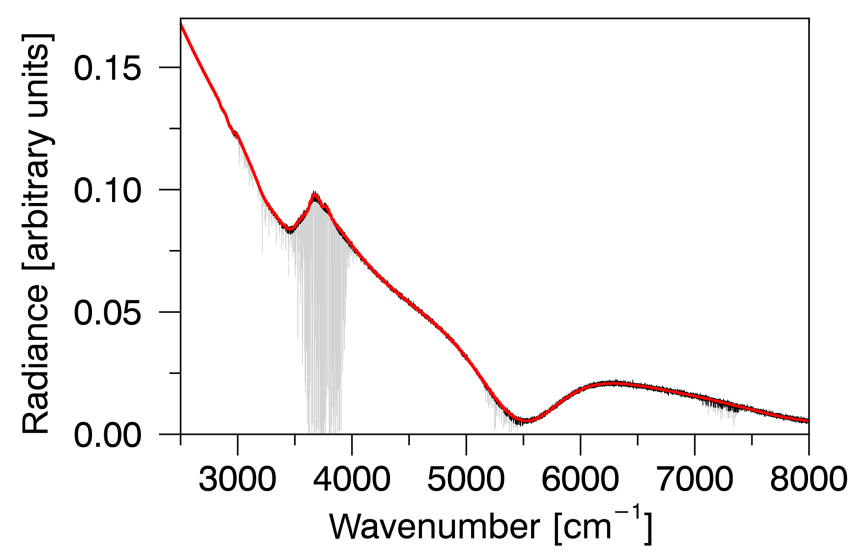

Figure 4. Blackbody radiance spectrum recorded on 24 February 2014 with a cavity temperature of $1923.15 \mathrm{~K}$. Grey: measured spectrum; black: result of spectral line exclusion; red: final spectrum after median filtering.

spectra were selected (black spectrum in Fig. 4). The influence of measurement noise can then be further reduced by applying a median filter $\left(20 \mathrm{~cm}^{-1}\right.$ width) to the spectra (red spectrum in Fig. 4). Finally, the blackbody calibration curve $c_{\mathrm{bb}}(v)$ is calculated from the averaged spectra by dividing the Planck curve at the cavity temperature $T_{\mathrm{bb}}$ by the measured spectrum. Standard lamps constitute an alternative to the high-temperature blackbody that can also be used in the combined calibration scheme. However, the spectral radiance of such calibration sources is typically prone to higher uncertainty than for blackbody sources.

\subsection{Combined calibration}

The combined calibration strategy takes advantage of the low-uncertainty Langley calibration at suitable spectral points (see Sect. 3.1). In between the Langley points, the shape of the calibration curve is constrained by the blackbody measurements (see Sect. 3.2). The combined calibration curve $c(v)$ is given by the relation

$c(v)=c_{\mathrm{bb}}(v) \cdot c_{\text {lan, linear }}(v) / c_{\mathrm{bb}, \text { linear }}(v)$,

where $c_{\mathrm{bb}}$ designates the blackbody calibration curve derived according to Sect. 3.2. $c_{\text {lan, linear }}$ and $c_{\mathrm{bb} \text {, linear }}$ designate linear interpolations constructed as follows: at the Langley calibration points, $c_{\mathrm{lan} \text {, linear }}$ and $c_{\mathrm{bb}}$, linear are set to the Langley and blackbody calibration results, respectively. In between these points, $c_{\text {lan, linear }}$ and $c_{\mathrm{bb} \text {, linear }}$ are calculated by linear interpolation. The combined calibration curve calculated according to Eq. (4) from blackbody measurements made on 24 February 2014 and Langley measurements made on 13 December 2013 is shown in Fig. 5.

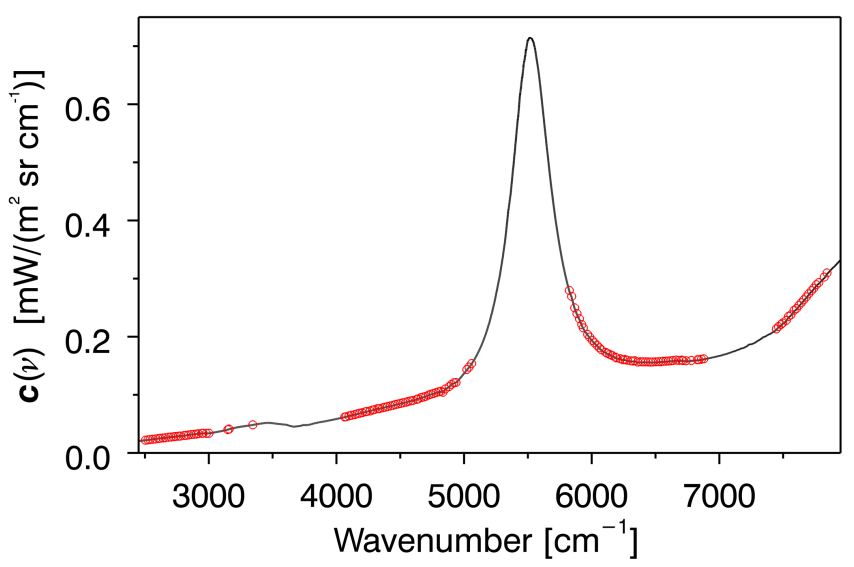

Figure 5. Combined calibration curve (black line) and selected Langley calibration points (red circles) for the Langley measurements made on 13 December 2013 in combination with blackbody measurements made on 24 February 2014.

\section{Calibration uncertainty}

\subsection{Contributions from Langley calibration}

Several contributions to the calibration uncertainty budget are associated with the Langley measurements. A first contribution results from the uncertainty of the Langley fit. This contribution is calculated as an error-weighted mean over the $2 \sigma$ uncertainties of the Langley calibration results for all spectral points contributing to each Langley point, i.e., it represents the uncertainty of the Langley extrapolation to air mass 0 . In between Langley points, the uncertainty estimate is obtained by linear interpolation between the uncertainty values at the Langley points. The Langley contribution to the calibration uncertainty ranges from 0.35 to $0.72 \%$ throughout the spectral range considered for calibration and is shown in Fig. 6 (blue line). The low uncertainty of the Langley fit is a result of the spectral point selection outlined in Sect. 3.1.3, which restricts the use of Langley results to spectral points with low fit uncertainty, while blackbody measurements are used to constrain the calibration curve in between these points. Note that, in addition to the Langley contribution, further uncertainty is induced by the combination with blackbody measurements between Langley points. This additional contribution leads to an increase in overall uncertainty between Langley points and will be outlined in Sect. 4.3.

Furthermore, the reflectivity of the solar tracker mirrors feature spatial inhomogeneity due to dirt and aging effects. Due to nonideal alignment of optical elements of the solar tracker, the area covered by the instrument's FOV of the tracker mirrors changes over time, i.e., depending on the azimuth and elevation of the instrument's line of sight. This leads to spurious radiance variations in the Langley calibration and increases the calibration uncertainty. To obtain an estimate of this error, the position of the instrument FOV 


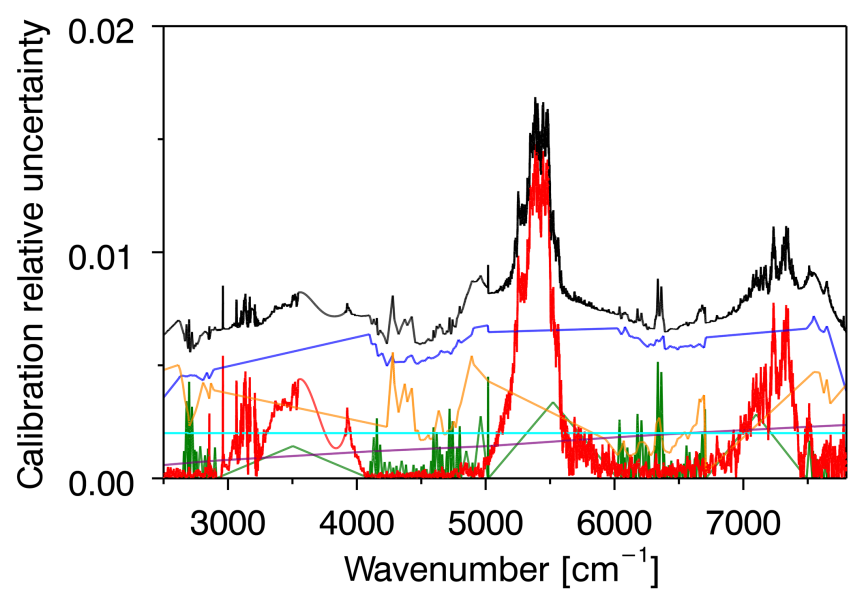

Figure 6. Relative $2 \sigma$ calibration uncertainty excluding the contribution due to ESS inaccuracies, which his discussed in Sect. 4.2 (Langley measurements made on 13 December 2013; blackbody measurements on 24 February 2014). Blue: Langley fit uncertainty; red: blackbody uncertainty; green: shape error uncertainty; orange: air mass uncertainty; purple: mispointing uncertainty; cyan: FOV uncertainty; black: total uncertainty relevant for radiative closure experiment (not including ESS contribution).

on the tracker elevation mirror for the azimuth and elevation values encountered during the Langley calibration has to be measured. This was achieved using an outgoing laser beam aligned with the instrument's optical axis, whose position on the tracker mirrors for a given azimuth and elevation is then monitored. In the spectral regions with least atmospheric absorption, the diurnal variation of the measured solar radiance is about $5 \%$. This variation is due to a combination of several contributions: a first contribution is due to the change in atmospheric optical depth (OD) with air mass as visible in the Langley plot in Fig. 2. In addition, other atmospheric effects such as temporally variable clouds contribute to the observed signal. A final contribution is due to the mirror-related effect mentioned above. A conservative estimate of the FOV-related error is obtained assuming that the observed diurnal variation is solely due to mirror inhomogeneity and that mirror reflectivity drops abruptly by this amount $(5 \%)$ outside the area initially covered by the FOV. Consequently, the error estimate is obtained by multiplying the $5 \%$ reflectivity change with the fraction by which the area within the field of view has changed throughout the time interval over which measurements contributing to the Langley fit were made. The interval is deduced from the laser measurements. The resulting Langley calibration uncertainty due to mirror inhomogeneity is $\sim 0.2 \%$ (cyan line in Fig. 6). Note that for the Zugspitze solar FTIR, the angle of incidence on the solar tracker mirrors does not vary significantly during measurements or in between blackbody and atmospheric measurements. Therefore, the angular dependence of mirror reflectivity is not included in the calibration uncertainty estimate. However, for different instruments, this contribution may have to be taken into account (see Gardiner et al., 2012).

The accuracy of the Langley results is also limited by errors in the air mass values used for the fit. Firstly, this is due to inaccurate solar zenith angle input. A second and by far dominant effect is due to the fact that the relative air mass for absorbing species with different concentration profiles is not equal for a given solar zenith angle. Depending on the spectral region, the dominant contribution to atmospheric OD for most Langley points is either due to water vapor or aerosols. For our analysis, water vapor relative air masses were used. The difference in the calibration results when performing the analysis with relative air columns instead of water vapor columns is up to $0.5 \%$ and was taken as an estimate of the air-mass-related calibration uncertainty (see orange line in Fig. 6).

An additional uncertainty contribution of up to $0.25 \%$ results from the uncertainty of the mispointing correction outlined in Sect. 3.1.3 (purple line in Fig. 6). This contribution includes two effects related to the mispointing uncertainty: the effects of air mass uncertainty in the Langley fit and the uncertainty in the solar limb darkening correction outlined in Sect. 3.1.3.

\subsection{ESS uncertainty contribution}

A further uncertainty contribution is associated with the ESS used in the Langley calibration. While no uncertainty estimate was provided by the authors for the spectrum of $\mathrm{Ku}$ rucz (2005), the $2 \sigma$ uncertainty of the Thuillier et al. (2003) spectrum is reported to be in the range of $1.2 \%$ at $4000 \mathrm{~cm}^{-1}$ to $1.8 \%$ at $8000 \mathrm{~cm}^{-1}$. Menang et al. (2013) state an uncertainty $(1 \sigma)$ of $3.3-6.0 \%$ for their ESS derived via the Langley method.

However, recent studies on the NIR ESS have yielded results which are partly inconsistent within the respective uncertainties and feature differences of up to $5-10 \%$ (see, e.g., Menang et al., 2013; Bolsée et al., 2014; Thuillier et al., 2014, 2015; Weber et al., 2015). The ongoing discussion about the magnitude of the ESS in the NIR implies that the ESS uncertainty estimates reported by recent studies may underestimate the real uncertainty. Therefore, the absolute radiometric uncertainty of the calibration scheme presented in this study remains tentative and more definite constraints require improved knowledge of the NIR ESS.

However, the ESS uncertainty only has a very minor influence on the main aim of this study, namely the use of calibrated solar FTIR spectra in a closure experiment for quantification of the NIR water vapor continuum. This important feature results from the fact that the same ESS is used for calibration and synthetic spectra calculation in the closure experiment and is demonstrated in the companion paper Part 3. Therefore, in the context of closure experiments, the relevant uncertainty budget does not include the ESS contribution and is shown in Fig. 6. 


\subsection{Uncertainty from blackbody measurements}

A further contribution to the calibration uncertainty results from the blackbody measurements. The blackbody calibration curve uncertainty was calculated as 2 times the standard deviation of all normalized blackbody calibration curves recorded under suitably dry atmospheric conditions (nearsurface atmospheric water vapor density $\rho_{\mathrm{H}_{2} \mathrm{O}}<1 \mathrm{~g} \mathrm{~m}^{-3}$ ). These measurements include spectra at $T_{\mathrm{bb}}=1923.15$ to $1973.15 \mathrm{~K}$ cavity temperature. The relative error in the combined calibration curve resulting from the uncertainty of the blackbody measurements does not exceed $1.5 \%$ and is shown in Fig. 6 (red lines).

\subsection{Combined calibration uncertainty estimate}

The combined calibration according to Eq. (4) is based on the assumption that the blackbody calibration curve between suitable Langley points can be approximately described by multiplying the Langley calibration curve with a linear function, i.e., that the following relation is fulfilled:

$c_{\mathrm{bb}}(v) / c_{\mathrm{bb}, \text { linear }}(v) \approx c_{\mathrm{lan}}(v) / c_{\text {lan, linear }}(v)$

Equation (5) is not exactly fulfilled if the reflectivity of the additional off-axis mirror used for blackbody measurements does not vary linearly between Langley points. As a consequence, an additional contribution to the calibration uncertainty results from the use of the combined approach presented in Sect. 3.3. In order to obtain an estimate of this shape error contribution, the following procedure was applied:

i. The calibration error induced by omitting the Langley result at each single Langley spectral point $v_{i}$ and using only the neighboring points $v_{i-1}$ and $v_{i+1}$ is calculated. A preliminary shape error curve is then constructed from the error values at all $v_{i}$ by linear interpolation.

ii. The final shape error estimate is set to 0 at all Langley points $v_{i}$ according to Eq. (4). At all spectral points halfway between Langley points, i.e., at $\left(v_{i+1}-v_{i}\right) / 2$, the final error estimate is set to the value of the preliminary curve determined in (i).

iii. At spectral points between those mentioned in (ii), the estimated error is calculated by linear interpolation.

The shape error generally increases with increasing Langley point spacing. By construction, the error estimate resulting from the method given above corresponds to a mean Langley point spacing 2 times as large as the real spacing. The error estimate provided above is therefore expected to overestimate the real errors in most cases. The final shape error estimate is shown as a green curve in Fig. 6 and is up to $0.5 \%$ throughout the spectral range considered. This low-uncertainty contribution also shows that the shape of the calibration curves derived from blackbody and Langley measurements is in good agreement. However, a comparison of the absolute calibration relying solely on blackbody measurements with Langley results is not feasible with the Zugspitze instrumental setup. This is due to the fact that for blackbody measurements signal losses due to the optics setup bias the absolute level of the blackbody calibration curve, which, however, does not influence the accuracy of the calibration with the combined method presented in this study.

Thermal emission from instrument parts at room temperature contributes a fraction of less than $10^{-5}$ to the measured solar radiance within the considered spectral interval. Contrary to the situation for instruments operating in the farinfrared spectral range, this contribution is therefore of negligible importance for the calibration error budget. Within the Zugspitze radiative closure experiment, the same ESS is used for Langley calibration and radiative transfer calculations. Therefore, the accuracy of the water vapor continuum optical depth derived in closure experiments from the calibrated spectra is not heavily affected by errors in the extraatmospheric solar spectrum. The overall uncertainty relevant for water vapor continuum detection, i.e., not including the extra-atmospheric solar spectrum contribution, is shown in Fig. 6 (black lines). It is typically below $1 \%$ within the calibration range, except for regions with sparse Langley point density or low beam splitter transmissivity where maxima of up to $1.7 \%$ uncertainty exist. The error budget including the uncertainty of the solar spectrum of Thuillier et al. (2003) is about $1.5-2 \%$ throughout the 4000 to $7800 \mathrm{~cm}^{-1}$ wavenumber range. However, as outlined in Sect. 4.2, the ESS uncertainty is a topic of ongoing debate and therefore the overall uncertainty budget should be treated as tentative and needs confirmation or adjustments by future ESS studies.

\section{Validation of results}

\subsection{Self-consistency}

A first method to validate the calibration results and the associated error estimate is to investigate the self-consistency of different calibration cases, i.e., the reproducibility of the calibration. The self-consistency of blackbody measurements is presented in Sect. 4.2. This result is then used as an estimate of the blackbody-related contribution to the total calibration uncertainty. As outlined in Sect. 4.2, this uncertainty contribution does not exceed $1.5 \%$ throughout the spectral interval considered for calibration. The reproducibility of the Langley results is estimated by comparing the Langley measurements made on 12 December 2013 with the ones made on 13 December 2013. As shown in the Fig. 7a, the calibration curves determined from those two Langley measurements typically differ by less than $1 \%$ outside absorption bands. In regions with sparse coverage of Langley points, i.e., within water vapor absorption bands, differences are typically around $1.5 \%$. 


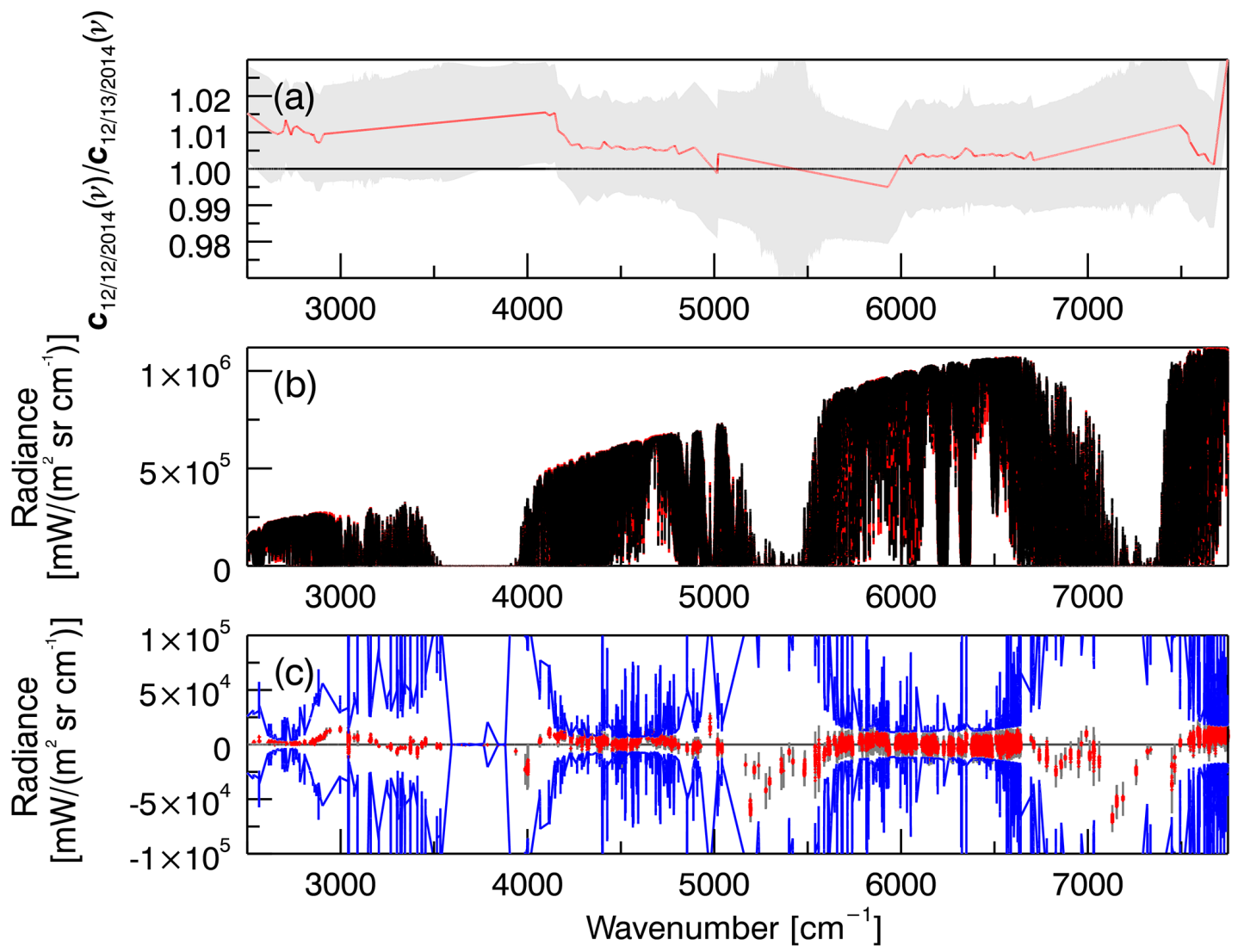

Figure 7. Examination of calibration results. (a) Ratio of the combined calibration curves determined from the 12 and 13 December 2013 Langley measurements in combination with the 24 February 2014 blackbody measurements (red line) and $2 \sigma$ uncertainty estimate (grey shaded area). (b) Mean measured (black) and synthetic (red) radiance for the validation data set. (c) Mean residual (red dots) and standard deviation of residuals (grey shaded area) for the validation data set outside spectral lines in comparison with the $2 \sigma$ residual uncertainty estimate (blue line).

Throughout $91.1 \%$ of the calibration spectral range (2500 to $7800 \mathrm{~cm}^{-1}$ ), the calibration curves are consistent within the calibration uncertainty estimate of Sect. 4 (grey shaded area in Fig. 7a). This extensive consistency consolidates the validity of the error budget presented in Sect. 4.

Note that modifications to the solar FTIR instrument such as realignment of optical elements requires repetition of the calibration procedure and the calibration results are only valid during periods with no significant change of instrument characteristics. Such changes can be detected, e.g., by monitoring the modulation efficiency of the FTIR or the instrumental line shape, which is achieved via routine $\mathrm{HCl}$ cell measurements (Hase et al., 2013). During the time interval covered by the measurements included in this study, no significant changes in instrument characteristics were detected.

Apart from modifications to the instrument which were discussed above, the accuracy of the radiometric calibration can decrease over time due to ice formation on the liquid nitrogen cooled InSb detector in the case of leaks in the detector's vacuum enclosure (see Gardiner et al., 2012). As outlined in the companion paper Part 3, the additional absorption by ice formation is most pronounced in the 3000 to $3400 \mathrm{~cm}^{-1}$ range and was estimated using lamp spectra routinely recorded with the solar FTIR. The maximum influence of ice formation on the measured radiance was $1.6 \%$ at $3200 \mathrm{~cm}^{-1}$ and was included in the uncertainty budget of the closure experiment.

\subsection{Comparison to model results}

A further consistency check of the calibration error estimate provided in Sect. 4 can be obtained by a closure of calibrated spectra with synthetic solar absorption spectra obtained by radiative transfer model calculations, which enables us to detect any large deviations of the real calibration accuracy from the uncertainty estimate given in Sect. 4. More specifically, a set of calibrated spectra is compared to synthetic spectra obtained with the LBLRTM_v12.2 radiative transfer model (Clough et al., 2005). The atmospheric state used as input to the line-by-line radiative transfer model (LBLRTM) calculations was determined as outlined in Part 1. In sum- 
mary, we use water vapor column data retrieved from the solar FTIR spectra. Water vapor profiles were set according to four-times-daily National Center for Environmental Prediction (NCEP) resimulation data, while for temperature profiles we used a combination of NCEP reanalysis results and a fitted near-surface profile obtained from FIR thermal emission spectra. $\mathrm{CO}_{2}, \mathrm{CH}_{4}$, and $\mathrm{N}_{2} \mathrm{O}$ column values were measured with the nearby Garmisch solar FTIR. Aerosol optical depth was constrained with sun photometer measurements.

For the closure analysis, we used spectra measured under clear-sky conditions during the December 2013 to February 2014 period, during which no realignment or other modifications to the spectrometer were performed. All spectra with an air mass greater than 9.0, i.e., a solar zenith angle greater than $\sim 84^{\circ}$, were discarded due to increasing inaccuracies in the ray tracing calculation and significant air mass variation during the spectral averaging period at high zenith angles. Additionally, the validation data set only includes spectra for which the radiance uncertainty due to FOV variations on the tracker mirrors (see Sect. 4.1) is negligible $(<0.1 \%)$. These selection thresholds lead to a validation data set of 52 spectra. For calibration, the 13 December 2013 Langley and 24 February 2014 blackbody results were used.

The corresponding synthetic spectra were then computed for all calibrated spectra in the validation data set. Figure $7 \mathrm{~b}$ shows the mean measured (black) and synthetic (red) radiance for this set of spectra. It illustrates the very good general level of agreement between calibrated and synthetic spectra. The mean spectral residuals, i.e., the difference between synthetic and measured radiance is shown in red in Fig. 7c, while the standard deviation of the residuals is shown in grey. Quantitatively accurate closure is only possible outside solar lines due to the high ESS uncertainty within the lines. We therefore exclude these regions from the comparison based on the selection criterion provided in Sect. 3.1.3. Within atmospheric lines, the uncertainty of the closure is dominated by atmospheric state and line parameter uncertainties and therefore does not provide substantial insights into the calibration accuracy. We therefore discarded these spectral points by excluding all spectral points below $99 \%$ of the upper envelope to the mean radiance in $20 \mathrm{~cm}^{-1}$ wide bins. As outlined in Sect. 3.2, the blackbody measurements demonstrate that the calibration curve varies only slowly with wavenumber and contains no narrow line-like features. Therefore, it is sufficient to validate the calibration results in suitable windows between spectral lines as outlined above. The residuals shown in Fig. 7 and their further quantitative analysis given hereafter refer to these suitable validation windows.

The accuracy of the calibration uncertainty provided in Sect. 4 can be assessed by comparing the mean spectral residuals to their estimated uncertainty (blue lines in Fig. 7c). In addition to the calibration uncertainty according to Sect. 4, the residual uncertainty given in Fig. 7 contains several further contributions. These contributions describe the atmospheric-state uncertainty and further contributions related to the solar FTIR spectral radiance measurements. A detailed assessment of this closure uncertainty budget is given in Part 1, Sect. 6. In addition to the contributions listed in Part 1, the uncertainty contribution associated with the water continuum absorption has to be taken into account. Since no uncertainty is provided for the MT_CKD 2.5.2 model (Mlawer et al., 2012) used in the synthetic spectra calculation, the continuum error estimate was set to the difference between the upper and lower end of continuum results provided by recent studies, namely the studies by Ptashnik et al. $(2012,2013)$ and the MT_CKD 2.5.2 model. A more detailed description of these data sets is given in Part 3.

As visible in Fig. 7c, the mean residuals show very good consistency with the estimated uncertainty. More specifically, a fraction of $97.7 \%$ of the residual values lies within the $2 \sigma$ residual uncertainty estimate. The mean residuals correspond to $1.2 \%$ of the measured radiance at each spectral point on average for the suitable comparison windows. This extensive agreement of the mean residuals with the uncertainty estimate further substantiates the calibration uncertainty budget presented in Sect. 4 . However, the closure analysis relies on an accurate representation and a comprehensive uncertainty budget of the atmospheric optical depth obtained via the LBLRTM calculations. This uncertainty budget is presented in detail in Sect. 6 of the companion paper Part 1. A comparison of the model results to the atmospheric OD derived directly from the Langley measurements shows very good agreement within the uncertainties as outlined in Sect. 4 of the companion paper Part 3. Note that since for both Langley calibration and model calculations the same extra-atmospheric solar spectrum is used, the closure analysis does not provide information on the accuracy of the ESS used. In addition to the calibration uncertainty, further sources of radiance uncertainty contribute in the closure setup, e.g., IWV uncertainty or uncertainties related to the water vapor continuum. Therefore, the closure analysis does not enable a full validation of the calibration uncertainty budget. Instead, the analysis provides an indication that the calibration uncertainty budget excluding the ESS contribution presented in Sect. 4 contains no major underestimation of the real uncertainty.

\section{Summary and conclusions}

We presented a novel radiometric calibration strategy for high-resolution solar FTIR spectral radiance measurements in the NIR and MIR which relies on a combination of the Langley method with radiance measurements of a blackbody source. While the Langley method yields highly accurate calibration results at a number of suitable spectral points, the blackbody measurements constrain the shape of the calibration curve in between these points. The combined calibration scheme therefore provides a $2 \sigma$ calibration uncertainty 
of about $1.0-1.7 \%$ throughout the spectral range employed for calibration ( 2500 to $7800 \mathrm{~cm}^{-1}$ ) excluding the contribution from ESS inaccuracies. As outlined in the companion paper Part 3, the ESS uncertainty contribution is only of very minor importance for the main aim of this study, i.e., the quantification of the NIR water vapor continuum in a closure experiment. For this specific aim, the achieved uncertainty constitutes a significant improvement compared to the uncertainty of $3.3-5.9 \%$ reached in previous studies. However, the absolute radiometric accuracy of the calibration scheme presented in this study (including the ESS contribution) has to be considered tentative due to the fact that the magnitude of the NIR ESS is a topic of ongoing debate. Future ESS studies are expected to resolve this issue and improve the reliability of the calibration presented in this study for general applications beyond closure experiments.

A central advantage of the combined method is that it provides sufficiently accurate calibration for the quantification of the NIR water vapor continuum in an atmospheric radiative closure experiment. Furthermore, the combined calibration scheme can be implemented also at remote sites including the Zugspitze summit observatory and therefore represents a suitable alternative to the method by Gardiner et al. (2012). However, contrary to the method by Gardiner et al. (2012), the combined method presented in this study is not directly traceable to a primary standard and its accuracy for applications beyond closure experiments relies on an accurate knowledge of the ESS, which, as outlined above, is a topic of ongoing research. Therefore, the presented method is currently best suited for the use in closure experiments, while future, more robust constraints on the NIR ESS are expected to provide the foundation for accurate low-uncertainty calibration with the combined method for other applications.

The calibration scheme was implemented in the spectral range of 2500 to $7800 \mathrm{~cm}^{-1}$. At lower wavenumber values, thermal emission from the instrument itself becomes non-negligible. Therefore, alternative methods such as the widely used method introduced by Revercomb et al. (1988) are more suitable for calibration in this spectral range. The proposed new method can, however, be implemented with minor changes in the spectral range beyond $7800 \mathrm{~cm}^{-1}$. Substitution of the solar FTIR detector and beam splitter (InGaAs detector and $\mathrm{CaF}_{2}$ beam splitter instead of $\mathrm{InSb} / \mathrm{KBr}$ ) allows for an extension of the calibration range up to about $9000 \mathrm{~cm}^{-1}$, while the use of a higher-temperature blackbody source or standard lamp enables calibration at even higher wavenumber values until the visible spectral range.

As outlined above, the use of a single blackbody calibration source is suitable for solar FTIR measurements in the NIR, contrary, e.g., to the Atmospheric Emitted Radiance Interferometer (AERI) (Knuteson et al., 2004) that achieves radiometric calibration in the FIR and MIR via the method proposed by Revercomb et al. (1988) using two blackbody sources at different cavity temperatures. This is mainly due to the negligible influence of thermal emission by the instru- ment on the measured radiance in the NIR (see Sect. 4.3). A nonlinear detector response represents a further issue that would require the use of multiple calibration sources. Eventual detector nonlinearity can be detected in the measured spectra as spurious radiance exceeding the measurement noise in saturated regions, i.e., within saturated spectral lines or in spectral regions beyond the detector's measurement range. However, using this method, no significant nonlinearity was found for the InSb detector setup used in this study. An extension of the proposed technique using an additional blackbody source at a different temperature is therefore useful when applying radiometric calibration to spectra in the wavenumber range below $2500 \mathrm{~cm}^{-1}$ or when using different detectors prone to significant nonlinearity. An assessment of the calibration uncertainty budget was made, containing contributions from the Langley fit, the blackbody measurements, the combination of both techniques in a single calibration curve, the solar tracker pointing accuracy, and the accuracy of the extra-atmospheric solar spectrum. The estimated uncertainty is below $1 \%$ in window regions and up to $1.7 \%$ within absorption bands. The calibration results are substantiated by the investigation of self-consistency for different calibration measurements and radiative closure with line-by-line model calculations. Both efforts indicate the validity of the $1.0-1.7 \%$ uncertainty estimate. Note, however, that the presented calibration method and the validity of the corresponding uncertainty estimate rely on a narrow range of atmospheric conditions, most notably the absence of clouds and low atmospheric water vapor content.

The presented scheme therefore fulfills the main goal, i.e., to provide sufficiently accurate radiometric calibration of solar FTIR spectra for the use in radiative closure experiments. Most notably, the calibration scheme thereby enables, for the first time, a quantification of the water vapor continuum in the NIR spectral range under atmospheric conditions, and the corresponding results are presented in the companion publication Part 3.

\section{Data availability}

The underlying data of Fig. 6 are available in the Supplement. Figures 2 to 5 and 7 are illustrations specific to the Zugspitze site. The underlying data can, however, be obtained at any time from the corresponding author on demand.

\section{The Supplement related to this article is available online at doi:10.5194/amt-9-4673-2016-supplement.}

Acknowledgements. We are grateful for the constructive and helpful reviews and short comments, which led to significant improvements of this paper. We furthermore thank H. P. Schmid 
(KIT/IMK-IFU) for his continual interest in this work. Funding by the Bavarian State Ministry of the Environment and Consumer Protection (contracts TLK01U-49581 and VAO-II TP I/01) and Deutsche Bundesstiftung Umwelt is gratefully acknowledged. We thank U. Köhler (Meteorologisches Observatorium Hohenpeißenberg, DWD) for providing ozone column measurements, M. Wiegner (LMU München) for access to sun photometer measurement data, P. Hausmann (KIT/IMK-IFU) for providing IWV retrievals, and F. Hase (KIT/IMK-ASF) for valuable discussions. Additionally, we are grateful for support by the Deutsche Forschungsgemeinschaft and the Open Access Publishing Fund of the Karlsruhe Institute of Technology.

The article processing charges for this open-access

publication were covered by a Research

Centre of the Helmholtz Association.

Edited by: H. Maring

Reviewed by: three anonymous referees

\section{References}

Bolsée, D., Pereira, N., Decuyper, W., Gillotay, D., Yu, H., Sperfeld, P., Pape, S., Cuevas, E., Redondas, A., Hernandéz, Y., and Weber, M.: Accurate Determination of the TOA Solar Spectral NIR Irradiance Using a Primary Standard Source and the Bouguer-Langley Technique, Sol. Phys., 289, 2433-2457, doi:10.1007/s11207-014-0474-1, 2014.

Borsdorff, T. and Sussmann, R.: On seasonality of stratomesospheric $\mathrm{CO}$ above midlatitudes: New insight from solar FTIR spectrometry at Zugspitze and Garmisch, Geophys. Res. Lett., 36, L21804, doi:10.1029/2009GL040056, 2009.

Burch, D. E.: Continuum absorption by $\mathrm{H}_{2} \mathrm{O}$. Report AFGL-TR81-0300, Air Force Geophys. Laboratory, Hanscom AFB, MA, USA, 1982.

Clough, S. A., Shephard, M. W., Mlawer, E. J., Delamere, J. S., Iacono, M. J., Cady-Pereira, K., Boukabara, S., and Brown P. D.: Atmospheric radiative transfer modeling: a summary of the AER codes, Short Communication, J. Quant. Spectrosc. Ra., 91, 233244, doi:10.1016/j.jqsrt.2004.05.058, 2005.

Delamere, J. S., Clough, S. A., Payne, V. H., Mlawer, E. J. Turner, D. D., and Gamache, R. R.: A far-infrared radiative closure study in the Arctic: Application to water vapor, J. Geophys. Res., 115, D17106, doi:10.1029/2009JD012968, 2010.

Gardiner, T. D., Coleman, M., Browning, H., Tallis, L., Ptashnik, I. V., and Shine, K. P.: Absolute high spectral resolution measurements of surface solar radiation for detection of water vapour continuum absorption, Philos. T. R. Soc. A, 370, 2590-2610, doi:10.1098/rsta.2011.0221, 2012.

Hase, F., Hannigan, J. W., Coffey, M. T., Goldman, A., Hopfner, M., Jones, N. B., Rinsland, C. P., and Wood, S. W.: Intercomparison of retrieval codes used for the analysis of high-resolution, ground-based FTIR measurements, J. Quant. Spectrosc. Ra., 87, 25-52, doi:10.1016/j.jqsrt.2003.12.008, 2004.

Hase, F., Drouin, B. J., Roehl, C. M., Toon, G. C., Wennberg, P. O., Wunch, D., Blumenstock, T., Desmet, F., Feist, D. G., Heikkinen, P., De Mazière, M., Rettinger, M., Robinson, J., Schneider, M., Sherlock, V., Sussmann, R., Té, Y., Warneke, T., and Weinzierl,
C.: Calibration of sealed $\mathrm{HCl}$ cells used for TCCON instrumental line shape monitoring, Atmos. Meas. Tech., 6, 3527-3537, doi:10.5194/amt-6-3527-2013, 2013.

Hausmann, P., Sussmann, R., and Smale, D.: Contribution of oil and natural gas production to renewed increase in atmospheric methane (2007-2014): top-down estimate from ethane and methane column observations, Atmos. Chem. Phys., 16, 3227-3244, doi:10.5194/acp-16-3227-2016, 2016.

Hestroffer, D. and Magnan, C.: Wavelength dependency of the Solar limb darkening, Astron. Astrophys., 333, 338-342, 1998.

Kämpfer, N.: Monitoring atmospheric water vapour, Ground-Based Remote Sensing and In-situ Methods, ISSI Scientific Report Series, Vol. 10, Springer, New York, USA, doi:10.1007/978-14614-3909-7, 2013.

Knuteson, R. O., Revercomb, H. E., Best, F. A., Ciganovich, N. C., Dedecker, R. G., Dirkx, T. P., Ellington, S. C., Feltz, W. F., Garcia, R. K., Howell, H. B., Smith, W. L., Short, J. F., and Tobin, D. C.: Atmospheric Emitted Radiance Interferometer. Part I: Instrument Design, J. Atmos. Ocean. Tech., 21, 1763-1776, doi:10.1175/JTECH-1662.1, 2004.

Kurucz, R. L.: The Solar Irradiance by Computation, available at: http://kurucz.harvard.edu/sun.html (last access: 16 September 2016), 2005.

Kurylo, M. J.: Network for the detection of stratospheric change, Proc. SPIE 1491, P. Soc. Photo-Opt. Ins., 168 (1 September 1991), doi:10.1117/12.46658, 1991.

Liou, K. N.: An Introduction to Atmospheric Radiation, Academic Press, Oxford, UK, 583 pp., doi:10.1256/003590003102695746, 2002.

Menang, K. P., Coleman, M. D., Gardiner, T. D., Ptashnik, I. V., and Shine, K. P.: A high-resolution near-infrared extraterrestrial solar spectrum derived from ground-based Fourier transform spectrometer measurements, J. Geophys. Res.-Atmos., 118, 53195331, doi:10.1002/jgrd.50425, 2013.

Mlawer, E. J., Payne, V. H., Moncet, J., Delamere, J. S., Alvarado, M. J., and Tobin, D.C.: Development and recent evaluation of the MT_CKD model of continuum absorption, Philos. T. R. Soc. A, 370, 1-37, doi:10.1098/rsta.2011.0295, 2012.

Paynter, D. J. and Ramaswamy, V.: An assessment of recent water vapor continuum measurements upon longwave and shortwave radiative transfer, J. Geophys. Res., 116, 2156-2202, doi:10.1029/2010JD015505, 2011.

Paynter, D. J. and Ramaswamy, V.: Investigating the impact of the shortwave water vapor continuum upon climate simulations using GFDL global models, J. Geophys. Res.-Atmos., 119, 1072010737, doi:10.1002/2014JD021881, 2014.

Paynter, D. J., Ptashnik, I. V., Shine, K. P., Smith, K. M., McPheat, R. A., and Williams, R. G.: Laboratory measurements of the water vapor continuum in the $1200-8000 \mathrm{~cm}^{-1}$ region between $293 \mathrm{~K}$ and $351 \mathrm{~K}$, J. Geophys. Res., 114, D21301, doi:10.1029/2008JD011355, 2009.

Ptashnik, I. V., McPheat, R. A., Shine, K. P., Smith, K. M., and Williams, R. G.: Water vapor self-continuum absorption in nearinfrared windows derived from laboratory measurements, J. Geophys. Res., 116, D16305, doi:10.1029/2011JD015603, 2011.

Ptashnik, I. V., McPheat, R. A., Shine, K. P., Smith, K. M., and Williams, R. G.: Water vapour foreign-continuum absorption in near-infrared windows from laboratory measurements, Philos. T. R. Soc. A, 370, 2557-2577, doi:10.1098/rsta.2011.0218, 2012. 
Ptashnik, I. V., Petrova, T. M., Ponomarev, Yu. N., Shine, K. P., Solodov, A. A., and Solodov, A. M.: Near-infrared water vapour self-continuum at close to room temperaturem J. Quant. Spectrosc. Ra., 120, 23-35, doi:10.1016/j.jqsrt.2013.02.016, 2013.

Rädel, G., Shine, K. P., and Ptashnik, I. V.: Global radiative and climate effect of the water vapour continuum at visible and nearinfrared wavelengths, Q. J. Roy. Meteor. Soc., 141, 727-738, doi:10.1002/qj.2385, 2015.

Reichert, A. and Sussmann, R.: The Zugspitze radiative closure experiment for quantifying water vapor absorption over the terrestrial and solar infrared - Part 3: Quantification of the mid- and near-infrared water vapor continuum in the 2500 to $7800 \mathrm{~cm}^{-1}$ spectral range under atmospheric conditions, Atmos. Chem. Phys., 16, 11671-11686, doi:10.5194/acp-16-11671-2016, 2016.

Reichert, A., Hausmann, P., and Sussmann, R.: Pointing errors in solar absorption spectrometry - correction scheme and its validation, Atmos. Meas. Tech., 8, 3715-3728, doi:10.5194/amt-83715-2015, 2015.

Revercomb, H. E., Buijs, H., Howell, H. B, LaPorte, D. D., Smith, W. L., and Sromovsk, L. A.: Radiometric calibration of IR Fourier transform spectrometers: solution to a problem with the High-Resolution Interferometer Sounder, Appl. Optics, 27, 3210-3218, doi:10.1364/AO.27.003210, 1988.

Schmid, B. and Wehrli, C.: Comparison of Sun photometer calibration by use of the Langley technique and the standard lamp, Appl. Optics, 34, 4500-4512, 1995.

Serio, C., Masiello, G., Esposito, F., Di Girolamo, P., Di Iorio, T., Palchetti, L., Bianchini, G., Muscari, G., Pavese, G., Rizzi, R., Carli, B., and Cuomo, V.: Retrieval of foreign-broadened water vapor continuum coefficients from emitted spectral radiance in the $\mathrm{H}_{2} \mathrm{O}$ rotational band from 240 to $590 \mathrm{~cm}^{-1}$, Opt. Express, 16, 15816-15833, doi:10.1364/OE.16.015816, 2008.

Sussmann, R. and Schäfer, K.: Infrared spectroscopy of tropospheric trace gases: combined analysis of horizontal and vertical column abundances, Appl. Optics, 36, 735-741, doi:10.1364/AO.36.000735, 1997.

Sussmann, R., Borsdorff, T., Rettinger, M., Camy-Peyret, C., Demoulin, P., Duchatelet, P., Mahieu, E., and Servais, C.: Technical Note: Harmonized retrieval of column-integrated atmospheric water vapor from the FTIR network - first examples for longterm records and station trends, Atmos. Chem. Phys., 9, 89878999, doi:10.5194/acp-9-8987-2009, 2009.

Sussmann, R., Forster, F., Rettinger, M., and Bousquet, P.: Renewed methane increase for five years (2007-2011) observed by solar FTIR spectrometry, Atmos. Chem. Phys., 12, 4885-4891, doi:10.5194/acp-12-4885-2012, 2012.

Sussmann, R., Reichert, A., and Rettinger, M.: The Zugspitze radiative closure experiment for quantifying water vapor absorption over the terrestrial and solar infrared - Part 1: Setup, uncertainty analysis, and assessment of far-infrared water vapor continuum, Atmos. Chem. Phys., 16, 11649-11669, doi:10.5194/acp-1611649-2016, 2016.
Thuillier, G., Hersé, M., Labs, D., Peetermans, W., Gillotay, D., Simon, P., and Mandel, H.: The solar spectral irradiance from 200 to $2400 \mathrm{~nm}$ as measured by the SOLSPEC spectrometer from the ATLAS and EURECA missions, Sol. Phys., 214, 1-22, doi:10.1023/A:1024048429145, 2003.

Thuillier, G., Bolsée, D., Schmidtke, G., Foujols, T., Nikutowski, B., Shapiro, A. I., Brunner, R., Weber, M., Erhardt, C., Hersé, M., Gillotay, D., Peetermans, W., Decuyper, W., Pereira, N., Haberreiter, M., Mandel, H., and Schmutz, W.: The Solar Irradiance Spectrum at Solar Activity Minimum Between Solar Cycles 23 and 24, Sol. Phys., 289, 1931-1958, doi:10.1007/s11207-0130461-y, 2014.

Thuillier, G., Harder, J. W., Shapiro, A., Woods, T. N., Perrin, J.M., Snow, M., Sukhodolov, T., and Schmutz, W.: The Infrared Solar Spectrum Measured by the SOLSPEC Spectrometer Onboard the International Space Station, Sol. Phys., 290, 15811600, doi:10.1007/s11207-015-0704-1, 2015.

Tobin, D. C., Best, F. A., Brown, P. D., Clough, S. A., Dedecker, R. G., Ellingson, R. G., Garcia, R. K., Howell, H. B., Knuteson, R. O., Mlawer, E. J., Revercomb, H. E., Short, J. F., van Delst, P. F., and Walden, V. P.: Downwelling spectral radiance observations at the SHEBA ice station: Water vapor continuum measurements from 17-26 $\mu \mathrm{m}$, J. Geophys. Res., 104, 2081-2092, doi:10.1029/1998JD200057, 1999.

Turner, D. D., Merrelli, A., Vimont, D., and Mlawer, E. J.: Impact of modifying the longwave water vapor continuum absorption model on community Earth system model simulations, J. Geophys. Res., 117, D04106, doi:10.1029/2011JD016440, 2012.

Vogelmann, H., Sussmann, R., Trickl, T., and Reichert, A.: Spatiotemporal variability of water vapor investigated using lidar and FTIR vertical soundings above the Zugspitze, Atmos. Chem Phys., 15, 3135-3148, doi:10.5194/acp-15-3135-2015, 2015.

Weber, M.: Comment on the Article by Thuillier et al. "The Infrared Solar Spectrum Measured by the SOLSPEC Spectrometer onboard the International Space Station”, Sol. Phys., 290, 16011605, doi:10.1007/s11207-015-0707-y, 2015.

Wunch, D., Toon, G. C., Blavier, J.-F. L., Washenfelder, R. A., Notholt, J., Connor, B. J., Griffith, D. W. T., Sherlock, V., and Wennberg, P. O.: The total carbon column observing network, Philos. T. Roy Soc. A, 369, 2087-2112, doi:10.1098/rsta.2010.0240, 2011. 